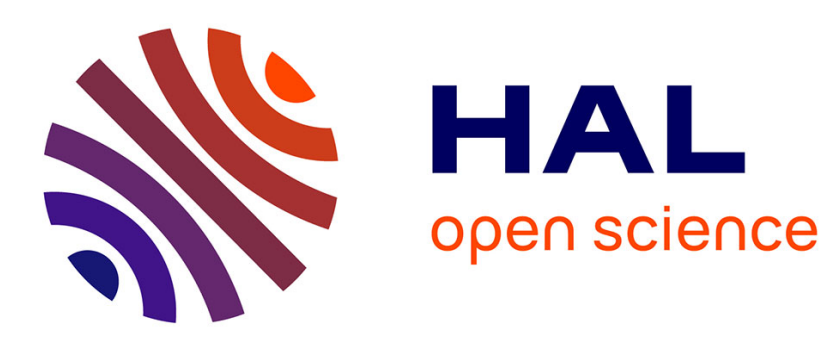

\title{
ÉTATS A GRAND NOMBRE DE QUASI-PARTICULE DANS LES ISOTOPES PAIRS DE PLOMB
}

\author{
G. Auger, V. Manfredi
}

\section{To cite this version:}

G. Auger, V. Manfredi. ÉTATS A GRAND NOMBRE DE QUASI-PARTICULE DANS LES ISOTOPES PAIRS DE PLOMB. Colloquium on Nuclear Surface, 1975, Dijon, France. pp.C5-91-C5-93, 10.1051/jphyscol:1975517 . jpa-00216372

\section{HAL Id: jpa-00216372 https://hal.science/jpa-00216372}

Submitted on 1 Jan 1975

HAL is a multi-disciplinary open access archive for the deposit and dissemination of scientific research documents, whether they are published or not. The documents may come from teaching and research institutions in France or abroad, or from public or private research centers.
L'archive ouverte pluridisciplinaire HAL, est destinée au dépôt et à la diffusion de documents scientifiques de niveau recherche, publiés ou non, émanant des établissements d'enseignement et de recherche français ou étrangers, des laboratoires publics ou privés. 


\title{
ÉTATS A GRAND NOMBRE DE QUASI-PARTICULE DANS LES ISOTOPES PAIRS DE PLOMB
}

\author{
G. AUGER et V. R. MANFREDI $\left(^{*}\right)$ \\ Institut de Physique Nucléaire, Division de Physique Nucléaire, \\ 91 Orsay, France
}

\begin{abstract}
Résumé. - Une étude par distribution spectrale dans les sous-espaces définis par leur nombre de quasi-particules a été effectuée dans les isotopes pairs de plomb. La comparaison avec des résultats obtenus dans les isotopes d'étain montre que le recouvrement entre les différents sous-espaces est fortement lié à l'interaction résiduelle utilisée. En particulier des états à grand nombre de quasiparticules sont présents à basse énergie. Le problème des états spurieux inhérent à cette méthode, qui sont responsables d'une surestimation du couplage, est abordé et différentes corrections sont proposées aussi bien sur les dimensions, les centroïdes et les largeurs des sous-espaces.
\end{abstract}

\begin{abstract}
The even lead isotopes have been studied by means of a spectral distribution calculation in the sub-spaces defined by their number of quasi-particles. The comparison with results obtained in the tin isotopes shows that the overlap of the various sub-spaces is strongly dependant on the residual interaction used; namely, states with a great number of quasi-particles do exist in the low energy part of the spectra. The problem of spurious states implied by this method, responsible for an over-estimation of the sub-space coupling, is treated and various corrections are proposed for the dimensions as well as for the centroids and widths of the sub-spaces.
\end{abstract}

Dans un article précédent [1], une application des méthodes de distribution spectrale dans les sousespaces définis par leur nombre de quasi-particules a été effectuée dans la région des isotopes pairs de l'étain. Les résultats les plus marquants de ces calculs sont : la présence d'états à grand nombre de quasiparticules à basse énergie, c'est-à-dire dans une région d'énèrgie où l'on admet généralement que les états sont préférentiellement décrits par un petit nombre de quasi-particules [2], ainsi que le fait que les couplages entre les différents sous-espaces de q-p sont très liés aux interactions résiduelles utilisées.

Dans le but d'extraire de ces résultats la partie liée à des propriétés générales (méthode des $q-p$, interaction résiduelle..., etc.) de celle liée à la structure particulière des isotopes étudiés (énergie des états individuels..., etc.), nous avons appliqué ces calculs aux isotopes pairs de plomb, où un modèle à deux quasiparticules reproduit d'une façon satisfaisante la forme générale des spectres à basse énergie [2].

Le calcul des quatre premiers moments associés aux spectres à deux quasi-particules obtenus par diagonalisation (pour ces isotopes) montre que les deux paramètres $\gamma_{1}$ et $\gamma_{2}$ (qui sont reliés au troisième et au quatrième moment de la distribution par $\gamma_{1}=\frac{\mu_{3}}{\sigma^{3}}, \quad \gamma_{2}=\frac{\mu_{4}}{\sigma^{4}}-3$ sont plus petits en valeur absolue que ceux observés dans la région des étains. $\mathrm{Ce}$ phénomène est essentiellement dû à la distri-

(*) Istituto di Fisica and Istituto Nationale di Fisica Nucleare, Sezione di Padova, Italia.• bution plus régulière des états individuels des isotopes de plomb $(\varepsilon 3 \mathrm{p} 1 / 2=0 ; \varepsilon$ 2f $5 / 2=0,54$; $\varepsilon$ 3p $3 / 2=0,9 ; \quad \varepsilon$ ii $13 / 2=1,63 ; \varepsilon$ ff $7 / 2=2,35$ en $\mathrm{MeV}$ ) comparée à celle asymétrique des étains $(\varepsilon 2 \mathrm{~d} 5 / 2=0 ; \quad \varepsilon \lg 7 / 2=0,2 ; \quad \varepsilon 3 \mathrm{~s} 1 / 2=2,1$; $\varepsilon 2 \mathrm{~d} 3 / 2=\varepsilon 1 \mathrm{~h} 11 / 2=3,2$ en $\mathrm{MeV}$ ). A la vue de ces valeurs qui restent en valeur absolue faibles, il semble justifié d'approximer les fonctions de fréquence associées aux différents sous-espaces par des fonctions gaussiennes (définies par les deux premiers moments de la distribution : $E$ la centroïde et $\sigma$ la variance).

En ce qui concerne les mélanges entre les différents sous-espaces de quasi-particules, nous avons retrouvé des résultats tout à fait similaires à ceux obtenus dans la région des étains. En effet, bien que les dimensions des sous-espaces, les énergies des états individuels et les paramètres des interactions soient différents, les recouvrements entre les différentes fonctions de fréquences associées aux sous-espaces de quasi-particules sont importants en ce qui concerne les forces du type gaussienne (True and Ford ou Wigner) et plus raisonnables pour des interactions tels la S.D.I. ou le Pairing, et ceci pour tous les moments angulaires. C'est ce que montrent les figures $1 a$ et $1 b$, où l'on s'est intéressé uniquement au domaine des basses énergies pour un noyau typique, le ${ }^{192} \mathrm{~Pb}$.

La conclusion que l'on peut tirer est qu'effectivement les mélanges des sous-espaces sont beaucoup plus importants qu'un calcul par diagonalisation pouvait le laisser prévoir; en particulier dans certains cas, 


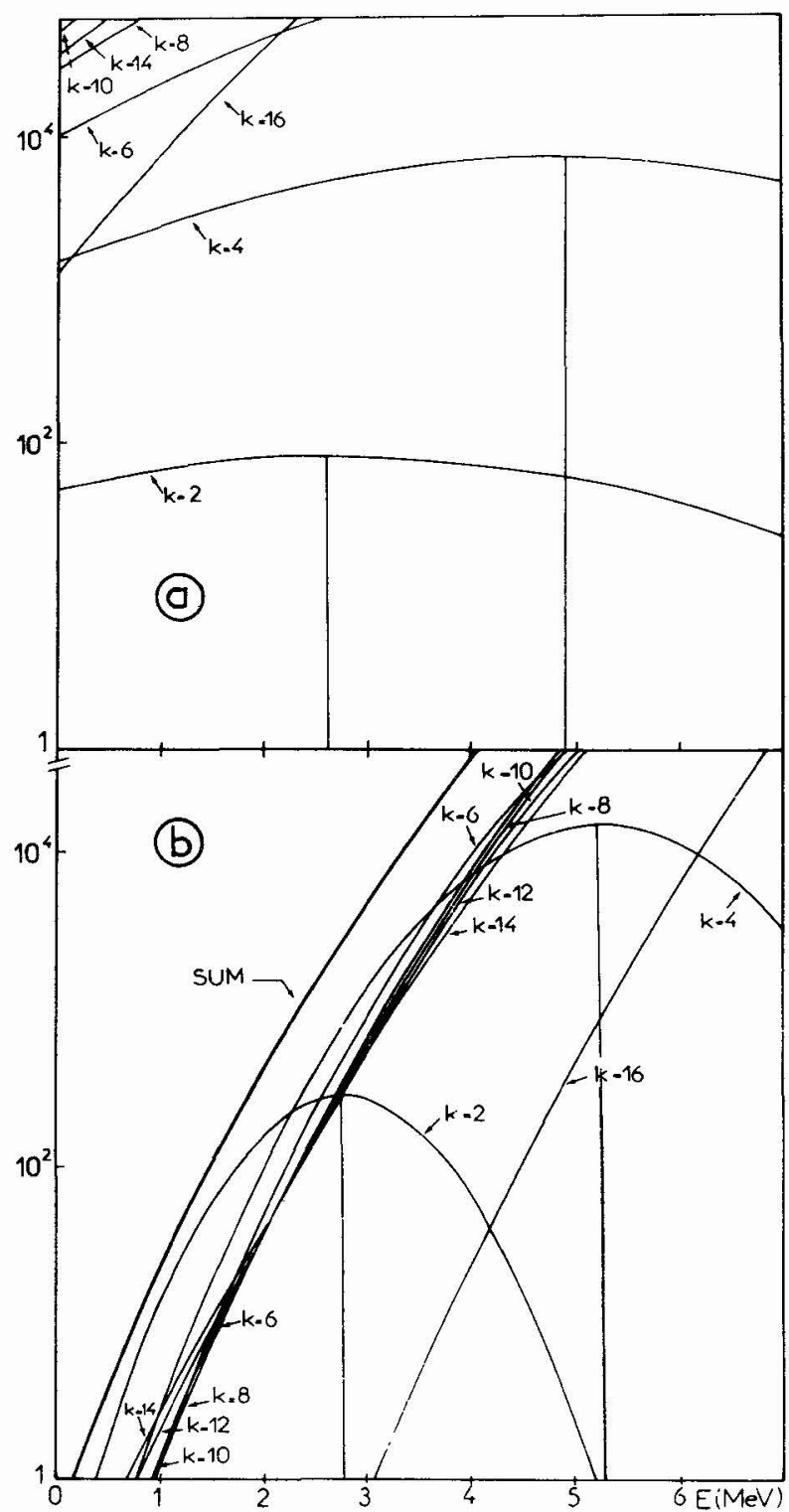

FIG. $1 a$ et $b .-$ Les fonctions de fréquence $f_{k}(E)$ sont montrées dans la région du centroide des états à deux q-p pour ${ }^{192} \mathrm{~Pb}$. La force utilisée est $: a$ ) force de Wigner, $b$ ) une S.D.I. (voir tableau I). Chaque fonction $f_{k}(E)$ est supposée gaussienne. Tous les sous-espaces étant couplés les uns aux autres suivant la règle $\sigma^{2}\left(k \rightarrow k^{\prime}\right) \neq 0$ si $-4<k^{\prime}<+4$. Les états non physiques sont compris.

même l'état de B.C.S. à des composantes à grand nombre de $q-p$, ces mélanges dépendant explicitement de l'interaction résiduelle utilisée.

Il n'empêche qu'une partie des recouvrements est certainement surestimée et ceci du fait de la présence d'états parasites directement liés aux méthodes de quasi-particules. A ce jour, nous ne savons pas calculer les différents moments dans des sous-espaces projetés sur le bon nombre d'états. Par contre, on peut proposer un certain nombre de corrections approximatives pour soustraire les états spurieux [1].

Le nombre d'états parasites dans le sous-espace à $k$ quasi-particules étant égal à $\left(\begin{array}{c}\Omega \\ k-\Omega\end{array}\right)$, la dimension corrigée est :

$$
\delta_{k}^{\prime}=\left(\begin{array}{l}
\Omega \\
k
\end{array}\right)-\left(\begin{array}{c}
\Omega \\
k-2
\end{array}\right)
$$

où $\Omega$ est le nombre d'états individuels.

Les largeurs corrigées :

$$
\sigma_{k}^{2 \prime}=\sigma^{2}(k \rightarrow k)+\sum_{k^{\prime} \neq k} \frac{\left(\begin{array}{l}
\Omega \\
k
\end{array}\right)-\left(\begin{array}{c}
\Omega \\
k-2
\end{array}\right)}{\left(\begin{array}{l}
\Omega \\
k
\end{array}\right)} \sigma^{2}\left(k \rightarrow k^{\prime}\right) .
$$

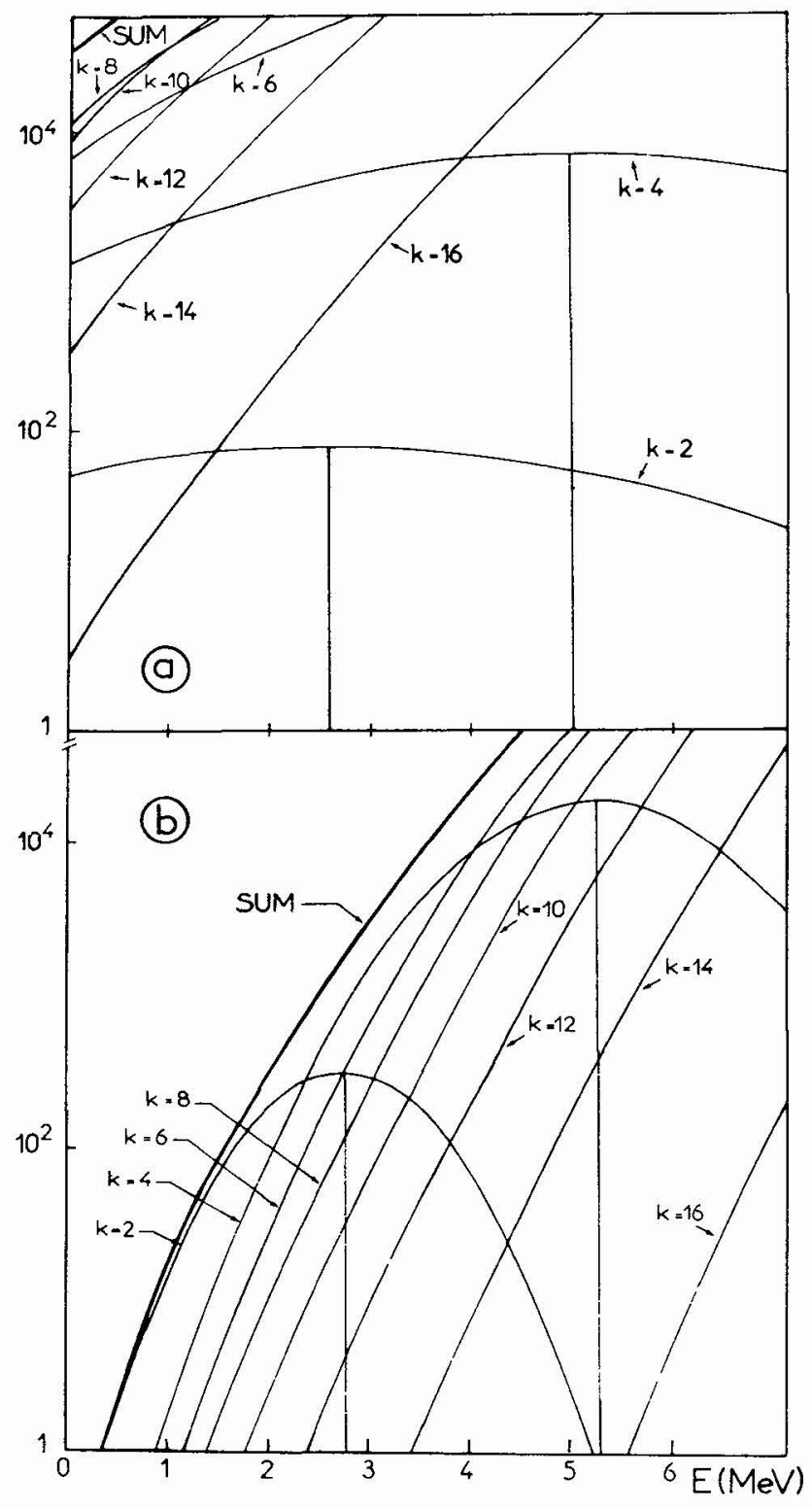

Fig. $2 a$ et $b$. - Cette figure diffère de la figure 1 par le fait que l'on a essayé d'extraire approximativement les états non physiques d'après les prescriptions du texte. 
Dans le même esprit, les centroïdes corrigées doivent s'écrire :

$$
\bar{E}_{k}^{\prime}=\frac{\left(\begin{array}{l}
\Omega \\
k
\end{array}\right) \bar{E}_{k}-\left(\begin{array}{c}
\Omega \\
k-2
\end{array}\right) \bar{E}_{k-2}}{\left(\begin{array}{l}
\Omega \\
k
\end{array}\right)-\left(\begin{array}{c}
\Omega \\
k-2
\end{array}\right)}
$$

En apportant ces différentes modifications, les effets des états à grand nombre de quasi-particules sont plus faibles comme on peut le voir sur la figure $2 a$ et $2 b$.

Pour essayer de voir si ces corrections sont suffisantes, nous avons comparé la largeur totale de tous les états excités d'un noyau, pour les états individuels considérés, obtenue de deux manières différentes; l'une où la largeur sera celle de la fonction de fréquence totale associée à la somme de toutes les fonctions de fréquences partielles de $q-p$

$$
F(E)=\sum_{k} f_{k}(E)
$$

L'autre obtenue d'après une formule établie par French [3] et donnant la largeur de tous les états de $m$ nucléons distribués dans $\Omega$ états individuels en fonc- tion de la largeur à un trou $\sigma^{2}(\Omega-1)$, de la largeur à une et deux particules $\sigma^{2}(1), \sigma^{2}(2)$.

$$
\begin{array}{r}
\sigma^{2}(m)=\frac{m(m-1)(m-2)(\Omega-m)(\Omega-m-1)}{(\Omega-1)(\Omega-2)(\Omega-3)} \times \\
\quad \times\left\{\frac{\sigma^{2}(\Omega-1)}{(\Omega-m-1)}-\frac{(\Omega-3)}{(m-1)} \sigma^{2}(1)+\frac{(\Omega-1)}{2(m-2)} \sigma^{2}(2)\right\} .
\end{array}
$$

Les résultats sont reportés tableau I où l'on a fait cette comparaison du ${ }^{204} \mathrm{~Pb}$ au ${ }^{192} \mathrm{~Pb}$. Il en ressort que les largeurs modifiées tendent effectivement à s'approcher des résultats de la formule exacte de French. Les divergences plus importantes que l'on obtient pour les noyaux à petit nombre de trous et donc décrits par un petit nombre de quasi particules montrent bien que cette façon d'exclure les états non physiques n'est pas suffisante. En effet, toutes ces corrections sont fonction du nombre d'états spurieux contenus dans le sous-espace, et pour $k$ petit ce pourcentage est très faible $(0,01 \%$ pour $k=4 \mathrm{q}-\mathrm{p})$. A l'inverse pour les états de $k$ grand ce pourcentage deviendra très élevé pour atteindre $63 \%$ pour $k=16 \mathrm{q}$-p.

En conclusion, il semble qu'il soit alors nécessaire de calculer explicitement les traces des opérateurs qui interviennent dans le calcul des moments dans des sous-espaces projetés sur les états physiques ce qui évidemment alourdit la méthode.

\section{TABleaU I}

Comparaison entre les largeurs obtenues d'après la somme des fonctions de fréquence associées aux différents sous-espaces de $\mathrm{q}-\mathrm{p}\left(F(E)=\sum_{k} f_{k}(E)\right)$ avec et sans correction, et d'après la formule de French
$($ calcul exact $)$

$\begin{array}{lcccccccc}A & 204 & 202 & 200 & 198 & 196 & 194 & 192 & 190 \\ \sigma_{\mathrm{qp}}^{2} \text { sans correction } & - & - & - & - & - & - & - & - \\ \sigma_{\mathrm{qp}}^{2} \text { avec correction } & 1,419 & 1,805 & 2,123 & 2,502 & 3,040 & 3,770 & 4,634 & 4,700 \\ \sigma^{2} \text { calcul exact } & 1,422 & 1,818 & 2,159 & 2,580 & 3,202 & 4,169 & 6,297 & 6,343 \\ & 2,114 & 3,187 & 4,182 & 5,042 & 5,725 & 6,199 & 6,441 & 6,441\end{array}$

\section{Bibliographie}

[1] Arvieu, R. et Manfredi, V. R., Nucl. Phys. A 239 (1975) 301.

[2] Pautrat, M., Thèse d'état, Paris (1972).

[3] French, J. B., Nucl. Structure (North Holland, Publ. Comp. Amsterdam) 1967. 